\title{
PD1/PD-L1 pathway in psoriasis and psoriatic arthritis: a review
}

\author{
Michał Adamczyk, Dorota Krasowska \\ Department of Dermatology, Venerology and Paediatric Dermatology, Medical University of Lublin, Lublin, Poland \\ Adv Dermatol Allergol 2021; XXXVIII (6): 925-930 \\ DOI: https://doi.org/10.5114/ada.2021.112274
}

\begin{abstract}
Programmed-death 1 (PD-1) is a co-receptor that inhibits the inflammatory response, and thus helps in maintenance of peripheral immunotolerance. Impairment in the PD-1/PD-L1 pathway is believed to play an important role in many immune-mediated diseases, including systemic lupus erythematosus, rheumatoid arthritis, systemic sclerosis and autoimmune hepatitis, and, as emphasized recently, in psoriasis and psoriatic arthritis. Biologic drugs targeting immune checkpoint regulators may be associated with new-onset psoriasis or exacerbations of pre-existing dermatosis. In this review we discuss the role of PD-1/PD-L1 pathway in psoriasis basing on data published to date.
\end{abstract}

Key words: psoriasis, psoriatic arthritis, PD-1/PD-L1, immune checkpoint.

\section{Introduction}

Psoriasis is a common, immune-mediated disease which manifests with erythematous papules and plaques with marked scaling on the skin. Psoriatic patients suffer from many co-morbidities including psoriatic arthritis, inflammatory bowel disease, autoimmune ocular disease, and most importantly metabolic syndrome with cardiovascular complications. In recent years all of the above has changed the understanding of psoriasis from only skin disease to the systemic condition associated with many severe complications and shortening of the life span [1].

Pathogenesis of psoriasis is complex and despite a lot of research conducted, still not fully understood. One of crucial phenomena in functioning of immune response in psoriasis is chronic T-cell activation by antigen presenting cells (APCs), but its exact cause remains unclear. Among many theories, a role of disruption in immuno-tolerance mechanisms is postulated as a part of development of psoriasis, as well as many other immune-mediated diseases [2]. In physiology, the natural immunotolerance is secured by various mechanisms, including central negative selection of autoreactive T-cells, peripheral anergy, peripheral suppression and clonal deletion [3].

The inhibitory co-receptor programmed death 1 (PD-1) is a glycoprotein expressed on T-cells, B lymphocytes, macrophages and monocytes. By interaction with its ligands on hematopoietic and non-hematopoietic cells, it blocks the activity of effector T-cells and promotes function of T-regulatory lymphocytes, being a well-known checkpoint inhibitor responsible for maintenance of peripheral self-tolerance [4].

Being so important in proper functioning of the immune system, it has been hypothesized that malfunctioning of PD-1 and/or its ligands may be of great importance in chronic hyperactivity of T-cell mediated immune response, which is observed in many different autoimmune conditions, including psoriasis. In this review we discuss the physiology and pathology of the PD-1/PD-L1 pathway and its possible role in psoriasis and psoriatic arthritis (PsA), basing on research published to date.

A search of databases was performed including PubMed and EMBASE using phrases: PD1, PD-L1, psoriasis and psoriatic arthritis. Overall, we found 14 studies evaluating the role of PD1/PD-L1 in psoriasis and psoriatic arthritis and all of them were included and analysed in this review (Figure 1).

\section{Physiological function of the PD-1/PD-L1 pathway}

PD-1, also known as CD279, is a transmembrane glycoprotein encoded by the PDCD1 gene and expressed on the surface of activated T-cells (most commonly CD4+),

Address for correspondence: Michał Adamczyk MD, Department of Dermatology, Venerology and Paediatric Dermatology, Medical University of Lublin, 11H Staszica St, 20-081 Lublin, Poland, phone: +48 693522 384, fax: +48 815323647 , e-mail: michaladamczyk1310@wp.pl Received: 2.06.2020, accepted: 19.07.2020. 


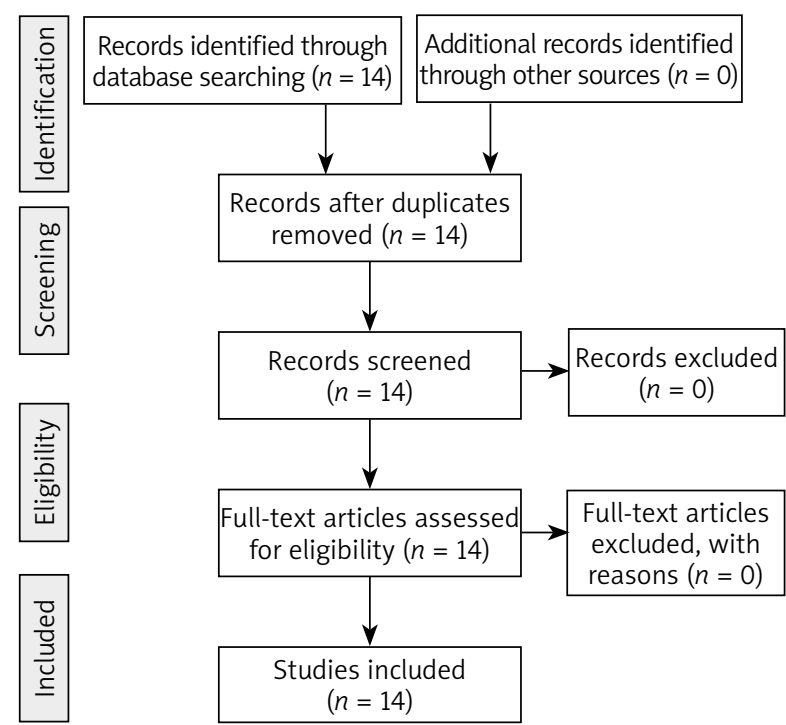

Figure 1. Flow diagram of the study search and selection process

B-cells and myeloid cells [5]. The molecule is a member of the CTLA-4 (cytotoxic T-lymphocyte-associated antigen 4, CD28) family and classified among $T$ cell regulators. The inhibitory effect on immune response is achieved by interaction between PD-1 and its ligands, PD-L1 and PD$\mathrm{L} 2$, which belong to the B7 family. PD-L1 is expressed on numerous hematopoietic and non-hematopoietic cells, like neutrophils, lymphocytes and additional nonimmune cells including tumours, endothelial cells, stromal cells and importantly, epithelial cells and keratinocytes [6]. PD-L2 is present on the surface of APCs [7, 8]. PD-L1 binds to PD1 and CD80, while PD-L2 binds only to PD-1. Interaction between PD-1 on CD4+ T-cells with CD80 located on APCs leads to reduction in CD4+ T cells activity, by inhibition of the phosphatidylinositol 3-kinase pathway, which results in decreased phosphorylation of Akt and reduced expression of GATA-3, T-bet and Eomes transcription factors. Another important result of PD-1/ PD1L ligation is a positive effect on $T$ regulatory lymphocyte (Tregs) quantity and function. Tregs are a subtype of T-cells responsible for suppression of immune response and maintenance of immunotolerance $[9,10]$. Concluding, signals mediated via PD-1 and its ligands are responsible for down-regulation of adaptive immune response, and PD-1 ligands expression by non-lymphoid cells like keratinocytes is important in maintaining immunotolerance in peripheral organs [11].

\section{PD-1/PD-L1 pathway in chronic inflammation}

In cases of chronic infections, neoplasms and autoimmune diseases, PD-1 is expressed in higher amounts by T-cells, and in these conditions it plays an important role in limiting protective immunity. The molecule is classi- fied among markers of T-cells activation [12]. PD-1 was found to be expressed at increased levels on T-cells from patients with systemic lupus erythematosus (SLE), rheumatoid arthritis (RA) and also with PSA $[13,14]$. Moreover, a few studies in RA patients confirmed increased frequencies of PD1+CD4+ T-cells in synovial fluid, when compared to peripheral blood and healthy controls [15].

As PD-1 is an inhibitory co-receptor, its increased expression in autoimmune diseases seems to be inconsistent. However, data from research provide evidence that under conditions of persistent chronic inflammation, the PD-1/PD-L1 pathway is modulated [15]. One of the studies demonstrated that T CD4+ T cells from synovial fluid from RA patients required higher concentrations of PDL1 to achieve inhibition when compared with peripheral blood T-cells [16]. In SLE, it was shown that two highly active pathways: Toll-like receptor (TLR) and type 1 interferon (IFN) regulate the expression of PD-1, PD-L1 and $\mathrm{PD}-\mathrm{L} 2$ via activation of NF- $\mathrm{KB}$ and STAT1, respectively [6].

Other important findings showed that soluble PD-1 (sPD-1) interfere with proper function of the PD-1/PD-L1 axis. SPD-1 binds to PD-L1 on the surface of peripheral cells and prevents proper PD-1/PD-L1 ligation, decreasing the inhibitory effect of the PD-1/PD-L1 pathway. The levels of SPD-1 are increased in chronic autoimmune conditions, like autoimmune hepatitis $(\mathrm{AH})$ and systemic sclerosis, RA and PSA $[17,18]$. In AH, the levels of SPD-1 correlated with disease severity and response to treatment [17], while in SSc they positively correlated with severity of skin sclerosis [18]. In patients with inflammatory arthritis like RA and PSA, the levels of SPD-1 were increased in both peripheral blood and synovial fluid [15].

Interestingly, it was shown in mice that disruption in the PDCD1 gene leads to various autoimmune conditions, like lupus-like syndrome, diabetes, proliferative arthritis, autoimmune cardiomyopathy and the increased risk of development of collagen-induced arthritis. What is in accordance with all above, the polymorphisms of PDCD1 gene in humans were associated with the increased risk of RA, SLE, type 1 diabetes mellitus, multiple sclerosis and ankylosing spondylitis [15]. Such predispositions have not been evaluated in psoriasis and psoriatic arthritis yet.

\section{PD-1/PD-L1 pathway and psoriasis}

According to the role of disruption of the PD-1/PD-L1 pathway in psoriasis, the data are quite sparse and some research presents contradictory results. Graphic presentation of data regarding the PD-1/PD-L1 pathway in Psoriatic disease published to date is shown in Figure 2, and summarized in Table 1. In all studies published to date small numbers of patients were engaged, not exceeding several dozens, so the results obtained should be verified in larger studies.

To begin with, there are numerous reports about new onset psoriasis or exacerbation of pre-existing psoriatic 


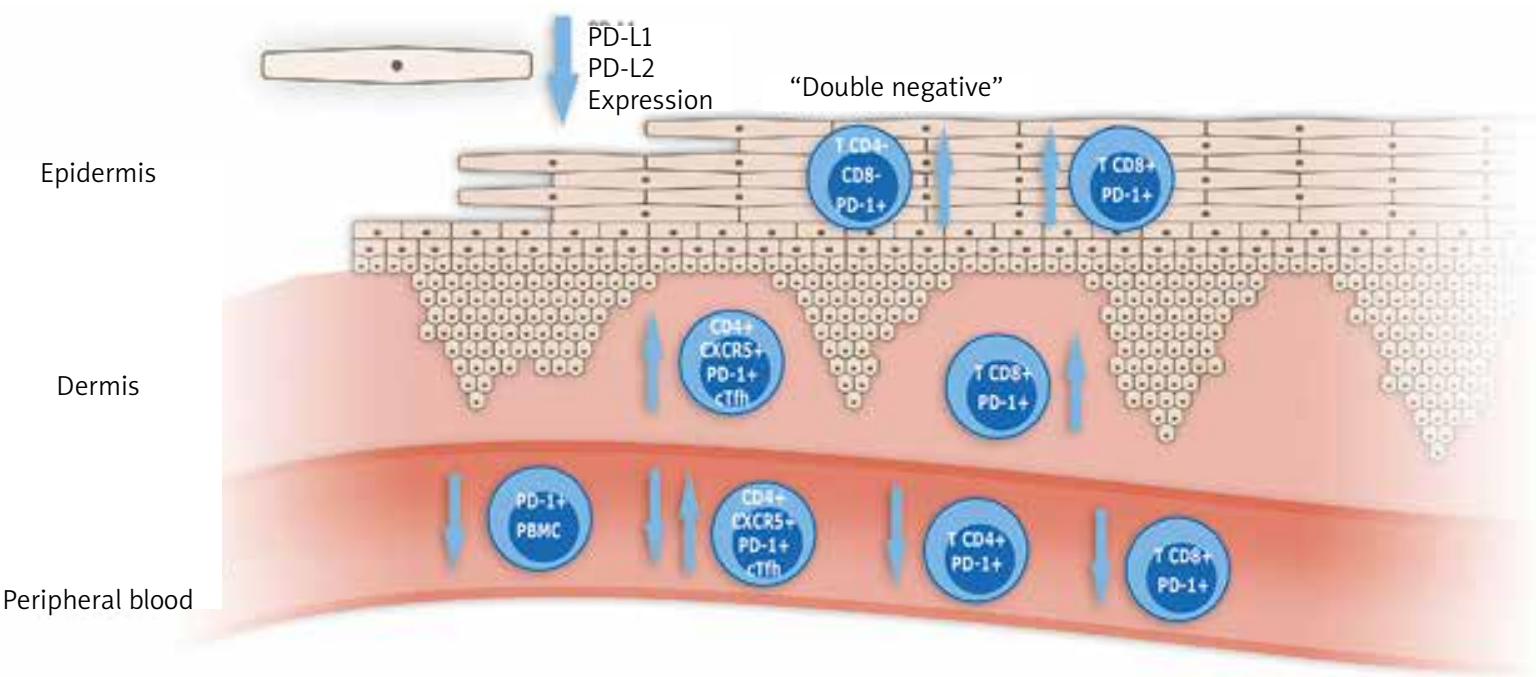

Figure 2. Graphic presentation of most important study results concerning PD-1/PD-L1 in patients with psoriasis and psoriatic arthritis. (cTfh - circulating T follicular helper cells, PBMC - peripheral blood mononuclear cells)

Table 1. A summary of most important data concerning the role of PD1/PD-L1 in psoriasis and psoriatic arthritis

\begin{tabular}{|c|c|c|}
\hline Finding & Possible significance & $\begin{array}{l}\text { Reference } \\
\text { number }\end{array}$ \\
\hline $\begin{array}{l}\text { PD-1 gene knock-out mice have greater keratinocyte hyperplasia, higher } \\
\text { expression of Th17 cytokines and more severe neutrophilic infiltrates in } \\
\text { skin lesions of imiquimod-induced psoriasis }\end{array}$ & $\begin{array}{l}\text { Role of PD-1 in limiting autoreactive response } \\
\text { in the murine model of psoriasis }\end{array}$ & 20 \\
\hline $\begin{array}{l}\text { Tonsil-derived mesenchymal stem cells transplanted into imiquimod- } \\
\text { induced psoriatic skin in mice exert a suppressive effect on Th17 } \\
\text { response via expression PD-L1 and stimulation of PD-1 expression on } \\
\text { immune cells }\end{array}$ & $\begin{array}{l}\text { Role of PD- } 1 \text { and PD-L1 in suppression of Th17 } \\
\text { cells in the murine model of psoriasis }\end{array}$ & 21 \\
\hline $\begin{array}{l}\text { Use of monoclonal antibodies against PD-1 and PD-L1 is associated with } \\
\text { a risk of new-onset psoriasis and exacerbation of pre-existing psoriatic } \\
\text { skin lesions }\end{array}$ & $\begin{array}{l}\text { Immune disruption caused by inhibition of } \\
\text { PD- } 1 \text { and PD-L1 results with enhancement of } \\
\text { inflammatory response }\end{array}$ & 19 \\
\hline $\begin{array}{l}\text { CD4+ T-cells derived from patients with psoriatic arthritis are resistant to PD- } 1 \\
\text { mediated suppression, which is probably caused by increased concentrations } \\
\text { of inflammatory cytokines (TNF- } \alpha \text {, IL- } 6 \text {, IL- } 1 \beta \text {, and soluble PD-1) }\end{array}$ & $\begin{array}{l}\text { Higher amounts of soluble PD-1, stimulated } \\
\text { by inflammatory cytokines is associated with } \\
\text { PD-1/PD-L1 disruption }\end{array}$ & 15 \\
\hline $\begin{array}{l}\text { The expression of PD-1 on circulating T-cells from patients with psoriatic } \\
\text { arthritis and in skin biopsies from psoriatic lesions is increased }\end{array}$ & PD-1 is a marker of T-cells activation & 14,22 \\
\hline $\begin{array}{l}\text { PD-1 positive follicular helper T cells are present in increased amounts } \\
\text { in peripheral blood from psoriatic patients, their levels correlate with } \\
\text { disease severity }\end{array}$ & $\begin{array}{l}\text { PD-1 positive follicular helper T cells may play } \\
\text { an important role in psoriatic inflammation }\end{array}$ & 26,27 \\
\hline $\begin{array}{l}\text { Double-negative, PD1+ T-cells infiltrate the epidermis in psoriatic skin } \\
\text { lesions }\end{array}$ & $\begin{array}{l}\text { Possible role in amplifying immune response } \\
\text { in psoriasis }\end{array}$ & 29 \\
\hline $\begin{array}{l}\text { Myeloid-derived suppressor cells are present in increased numbers in } \\
\text { peripheral blood of patients with psoriasis, but have lower expression } \\
\text { levels of PD-1 and PD-L1 }\end{array}$ & $\begin{array}{l}\text { Myeloid-derived suppressor cells in psoriasis } \\
\text { cannot suppress autologous and heterologous } \\
\text { CD8+ T-cell proliferations and fail to produce } \\
\text { immuno-competent Tregs }\end{array}$ & 30 \\
\hline $\begin{array}{l}\text { Expression of PD-L1 and PD-L2 in psoriatic epidermis is significantly } \\
\text { decreased when compared with healthy epidermis }\end{array}$ & $\begin{array}{l}\text { The lack of PD-1 ligands may lead to } \\
\text { a decrease in Treg activity and up-regulation } \\
\text { of T-cell mediated inflammatory response }\end{array}$ & 31 \\
\hline
\end{tabular}

lesions in patients treated with biologic drugs targeting the PD-1/PD-L1 pathway and antagonizing its function (so-called immune checkpoint inhibitors) [19]. They concerned monoclonal antibodies targeting both PD-1 (nivolumab, pembrolizumab) and PD-L1, which due to disruption in PD-1 ligation interfere with proper inhibitory function of the PD-1/PD-L1 pathway and enhance immune response. Such effect is highly desirable in the 
treatment of malignancies, but unfortunately results in the increased risk of many different autoimmune adverse events, including psoriasis. Except for psoriasis, other skin-related adverse events are also quite frequent in this group of patients, and include maculopapular rashes, lichenoid dermatitis, pruritus, acneiform lesions and urticaria. What should be emphasized is that most skinrelated side effects are mild and can be easily managed with topical drugs, so they do not require treatment cessation, but early treatment intervention is necessary to avoid it [19].

Imai et al. performed an interesting study, in which they compared PD-1 gene knock-out (KO) mice with wild-type mice in an imiquimod-induced murine model of psoriasis. PD-1 KO mice presented greater keratinocyte hyperplasia, higher expression of Th17 cytokines and more severe neutrophilic infiltrates when compared to wild-type mice. Moreover, $\mathrm{KO}$ mice showed 3-fold higher amounts of IL-17 expressing $T \gamma \delta$ cells in skin cell suspensions [20]. These findings support the role of PD-1 in limiting autoreactive response in the murine model of psoriasis.

In another experimental study Kim et al. evaluated the impact of tonsil-derived mesenchymal stem cells (T-MSCS) on imiquimod induced psoriatic dermatitis in mice. T-MSC express high levels of PD-L1, both transmembrane and soluble forms, in addition they stimulate the expression of PD-1 on T-cells by secretion of IFN- $\beta$. So this subset of MSC exerts a suppressive impact on immune response by promoting the PD-1/PD-L1 pathway. The au- thors demonstrated that transplantation of T-MSCs into imiquimod-induced psoriatic skin inflammation substantially decreased disease symptoms, mostly by decreasing Th17 immune response in a PD-L1-dependent way [21]. Their findings proved indirectly a role of the disrupted PD-1/PD-L1 pathway in psoriatic skin inflammation.

Bommarito et al. made an attempt to evaluate the impact of inflammation in PSA and RA patients on PD-1 mediated suppression of T-cells, and to clarify what exactly this process is influenced by. They examined mononuclear cells in peripheral blood (PB) and synovial fluid (SF). Flow cytometry showed an increased expression of PD-1 in RA and PsA patients on both CD4+ and CD8+ Tcells in SF and PB, which was higher in SF. What is more, they found that CD4+ T-cells derived from patients who were resistant to PD-1 mediated suppression, and this effect was probably caused by increased concentrations of inflammatory cytokines, such as TNF- $\alpha, I L-6, I L-1 \beta$, and, interestingly soluble PD-1 (sPD-1), which amounts were also elevated in PsA and RA subjects. They proved this finding in in vitro studies. Moreover, inhibitors of abovementioned cytokines reversed this effect. The authors demonstrated that IL-1 $\beta$, IL- 6 and TNF- $\alpha$ increased the levels of sPD-1 [15]. Higher expression of sPD-1which binds to PD ligands, enhanced by inflammatory cytokines results in disruption of the PD-1/PD-L1 pathway. No studies published to date evaluated concentrations of SPD-1 in psoriatic plaque environment. The modulation of PD-1/PD-L1 ligation by inflammatory cytokines is shown in Figure 3.

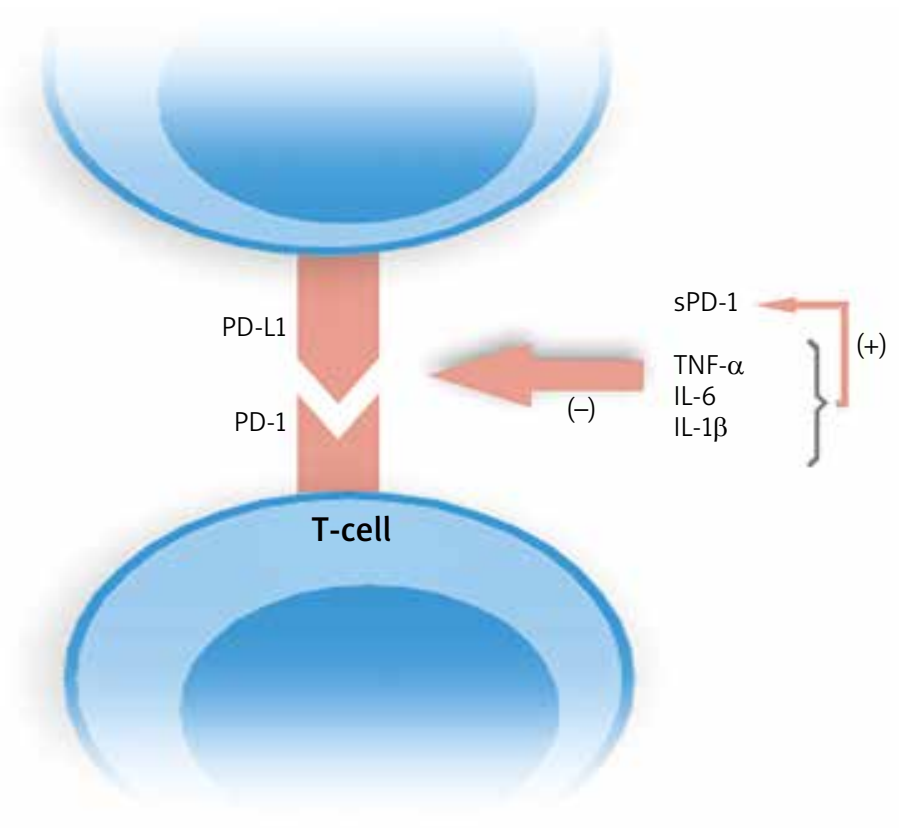

Figure 3. Modulation of PD1/PD-L1 ligation by inflammatory cytokines and SPD1 
Çetinözman compared the expression of PD1 on Tcells in skin biopsies taken from erythrodermic patients with skin lymphomas and inflammatory conditions, including 6 patients with psoriatic erythroderma. In biopsies from psoriatic lesions they found increased levels of PD-1 on CD8+ T-cells in epidermis and dermis [22]. Since PD-1 is a marker of T-cells activation, these results are not surprising.

Peled et al. evaluated the expression of PD-1 on circulating T-cells from patients with psoriatic arthritis and a healthy control group. They demonstrated that expression levels of PD-1 were significantly higher in PsA than in healthy controls, moreover they inversely correlated with disease activity measured by DAS28. Also, a strong correlation between PD-1 expression and a number of tender and swollen joints was found [14].

On the contrary to previously mentioned research, Bartosińska et al. reported down regulated expression of the PDCD1 gene in peripheral blood mononuclear cells (PBMCS) obtained from patients with psoriasis when compared to a healthy control, but no difference in the expression of the aforementioned gene between patients with psoriasis and psoriatic arthritis [23]. The same authors in their previous research demonstrated that absolute numbers and percentages of CD4+PD-1+ and CD8+PD-1+ T cells in PB of psoriatic patients both with and without PsA were significantly decreased when compared to healthy individuals [24, 25].

Recent studies evaluated several specific subtypes of T-cells in patients with psoriasis and psoriatic arthritis. Follicular helper $T$ cells (Tfh) are a specialized subset of CD4+ T-cells located in germinal centres, which express PD-1, chemokine CXC receptor 5 (CXCR5), inducible T cell costimulator (ICOS), and the transcription factors $B$ cell lymphoma 6 (Bcl-6) and secrete IL-21. Their most important role is to help in activation and production of antibodies by B cells. Circulating Tfh cells (cTfh) and their dysregulated function were proved to play a role in autoimmune diseases, including SLE, RA and infections, like tuberculosis and HIV infection [26, 27]. Niu et al. reported increased amounts of circulating Tfh cells in peripheral blood from psoriatic patients, moreover, their levels correlated with disease severity measured by Psoriasis Area and Severity Index (PASI) [27]. Wang et al. evaluated the presence of cTfh in peripheral blood (PB) and lesional skin (LS) from psoriatic patients. Like previously mentioned researchers, they found that numbers of PD-1 positive cTfh cells in PB were significantly higher in psoriatic patients than in healthy controls, and in lesional skin biopsies the amounts of cTfh were higher than in healthy epidermis. Higher frequency of cTfh cells was positively associated with PASI score, and, interestingly after treatment with acitretin the levels of cTfhs significantly decreased [26]. Conversely, Shin et al. reported decreased levels of cTfh cells in psoriatic patients, they also did not find any correlation between cTfh amounts and PASI [28].
Another interesting subtype of T cells is double-negative (DN) T-cells (CD4-/CD8-), which originate from CD8+ $T$ cells via down-regulation of CD8. Majority of them demonstrate a memory effector phenotype, they are also characterized by increased expression of PD-1, decreased DNA methylation of IFNG gene and thus expression of interferon $\gamma$. Recently, it was demonstrated that PD-1+ DN T-cells infiltrate the epidermis in skin lesions, suggesting an important role of this new subset of T-cells in amplifying immune response in psoriasis. Further research is needed to fully clarify their role [29].

Myeloid-derived suppressor cells (MDSC) were recently demonstrated to have an important role in cancer and immune-mediated diseases. Cao et al. reported increased numbers of circulating monocytic(Mo)-MDSC and demonstrated that in psoriasis patients they cannot suppress autologous and heterologous CD8+ T-cell proliferations, have lower expression levels of PD-1 and PD-L1, and fail to produce immuno-competent Tregs [30]. Decreased expression of PD-1 and PD-L1 may be implicated in disrupted function of MDSC in psoriasis.

According of PD-1 ligands in psoriatic epidermis, Kim et al. evaluated the expression of PD-L1 and PD-L2 in Psoriatic epidermis on mRNA and protein levels and found that it was significantly decreased when compared with healthy epidermis. The lack of PD-1 ligands may lead to a decrease of Treg activity which is typical for psoriasis and up-regulation of T-cell mediated inflammatory response, emphasizing the role of keratinocytes as one of the key components that amplify and maintain chronic inflammation in psoriasis [31].

\section{Conclusions}

Evidence supports the role of the PD-1/PD-L1 axis disruption in psoriatic patients, however data are quite sparse and presented study results are often inconsistent. Several important aspects, like the exact role of SPD-1 and other factors in PD-1/PD-L1 pathway modulation, genetic polymorphisms of PDCD1, altered expression of PD-1 ligands await to be carefully evaluated in psoriatic patients. In the future, medications modulating PD-1/PD-L1 pathway function may be another promising option in therapy of psoriasis and other autoimmune diseases.

\section{Conflict of interest}

The authors declare no conflict of interest.

\section{References}

1. Grozdev I, Korman N, Tsankov N. Psoriasis as a systemic disease. Clin Dermatol 2014; 32: 343-50.

2. Deng Y, Chang C, Lu Q. The inflammatory response in psoriasis: a comprehensive review. Clin Rev Allergy Immunol 2016; 50: 377-89. 
3. Bardhan K, Anagnostou T, Boussiotis VA. The PD1:PD-L1/2 pathway from discovery to clinical implementation. Front Immunol 2016; 12: 550.

4. Chen Z, Pang N, Du R, et al. Elevated expression of programmed death-1 and programmed death ligand-1 negatively regulates immune response against cervical cancer cells. Mediators Inflamm 2016; 2016: 6891482.

5. Agata Y, Kawasaki A, Nishimura $H$, et al. Expression of the PD-1 antigen on the surface of stimulated mouse $T$ and $B$ lymphocytes. Int Immunol 1996; 8: 765-72.

6. Curran CS, Gupta S, Sanz I, Sharon E. PD-1 immunobiology in systemic lupus erythematosus. J Autoimmun 2019; 97: 1-9.

7. Freeman GJ, Long AJ, Iwai Y, et al. Engagement of the PD-1 immunoinhibitory receptor by a novel B7 family member leads to negative regulation of lymphocyte activation. J Exp Med 2000; 192: 1027-34.

8. Latchman Y, Wood CR, Chernova T, et al. PD-L2 is a second ligand for PD-1 and inhibits T cell activation. Nat Immunol 2001; 2: 261-8.

9. Parry RV, Chemnitz JM, Frauwirth KA, et al. CTLA-4 and PD-1 receptors inhibit $\mathrm{T}$-cell activation by distinct mechanisms. Mol Cell Biol 2005; 25: 9543-53.

10. Kao C, Oestreich KJ, Paley MA, et al. Transcription factor Tbet represses expression of the inhibitory receptor PD-1 and sustains virus-specific CD81 T cell responses during chronic infection. Nat Immunol 2011; 12: 663-71.

11. Butte MJ, Keir ME, Phamduy TB, et al. Programmed death-1 ligand 1 interacts specifically with the B7-1 costimulatory molecule to inhibit T cell responses. Immunity 2007; 27 : 111-22.

12. Sharpe AH, Pauken KE. The diverse functions of the PD1 inhibitory pathway. Nat Rev Immunol 2018; 18: 153-67.

13. Okazaki T, Wang J. PD-1/PD-L pathway and autoimmunity. Autoimmunity 2005; 38: 353-7.

14. Peled M, Strazza M, Azoulay-Alfaguter I, et al. Analysis of programmed death-1 in patients with psoriatic arthritis. Inflammation 2015; 38: 1573-9.

15. Bommarito D, Hall C, Taams LS, Corrigall VM. Inflammatory cytokines compromise programmed cell death-1 (PD-1)-mediated T cell suppression in inflammatory arthritis through up-regulation of soluble PD-1. Clin Exp Immunol 2017; 188 : 455-66.

16. Raptopoulou AP, Bertsias G, Makrygiannakis D, et al. The programmed death 1/programmed death ligand 1 inhibitory pathway is up-regulated in rheumatoid synovium and regulates peripheral T cell responses in human and murine arthritis. Arthritis Rheum 2010; 62: 1870-80.

17. Aarslev K, Dige A, Greisen SR, et al. Soluble programmed death-1 levels are associated with disease activity and treatment response in patients with autoimmune hepatitis. Scand J Gastroenterol 2016; 52: 93-9.

18. Yanaba K, Hayashi M, Yoshihara Y, Nakagawa H. Serum levels of soluble programmed death-1 and programmed death ligand-1 in systemic sclerosis: association with extent of skin sclerosis. J Dermatol 2016; 43: 954-7.

19. Sibaud V, Meyer N, Lamant L, et al. Dermatologic complications of anti-PD-1/PD-L1 immune checkpoint antibodies. Curr Opin Oncol 2016; 28: 254-63.

20. Imai Y, Ayithan N, Wu X, et al. Cutting edge: PD-1 regulates imiquimod-induced psoriasiform dermatitis through inhibition of IL-17A expression by Innate $\gamma \delta$-low T cells. J Immunol 2015; 195: 421-5.

21. Kim JY, Park M, Kim YH, et al. Tonsil-derived mesenchymal stem cells (T-MSCS) prevent Th17-mediated autoimmune response via regulation of the programmed death-1/programmed death ligand-1 (PD-1/PD-L1) pathway. J Tissue Eng Regen Med 2018; 12: e1022-33.

22. Çetinözman F, Jansen PM, Willemze R. Expression of programmed death-1 in skin biopsies of benign inflammatory vs. lymphomatous erythroderma. Br J Dermatol 2014; 171: 499-504.

23. Bartosińska J, Purkot J, Kowal M, et al. The expression of selected molecular markers of immune tolerance in psoriatic patients. Adv Clin Exp Med 2018; 27: 721-5.

24. Bartosińska J, Zakrzewska E, Raczkiewicz D, et al. Suppressed programmed death 1 expression on CD4+ and CD8+ T cells in psoriatic patients. Mediators Inflamm 2017; 2017: 5385102.

25. Bartosińska J, Zakrzewska E, Purkot J, et al. Decreased blood CD4+PD-1+ and CD8+PD-1+ T cells in psoriatic patients with and without arthritis. Adv Dermatol Allergol 2018; 35: 34450.

26. Wang Y, Wang L, Yang $H$, et al. Activated circulating $T$ follicular helper cells are associated with disease severity in patients with psoriasis. J Immunol Res 2016; 2016: 7346030.

27. Niu J, Song Z, Yang X, et al. Increased circulating follicular helper $T$ cells and activated B cells correlate with disease severity in patients with psoriasis. J Eur Acad Dermatol Venereol 2015; 29: 1791-6.

28. Shin D, Kim DS, Kim SH, et al. Decreased PD-1 positive blood follicular helper T cells in patients with psoriasis. Arch Dermatol Res 2016; 308: 593-9.

29. Brandt D, Sergon M, Abraham S, et al. TCR+CD3+CD4-CD8effector T cells in psoriasis. Clin Immunol 2017; 181: 51-9.

30. Cao LY, Chung JS, Teshima T, et al. Myeloid-derived suppressor cells in psoriasis are an expanded population exhibiting diverse T-cell-suppressor mechanisms. J Invest Dermatol 2016; 136: 1801-10.

31. Kim DS, Je JH, Kim SH, et al. Programmed death-ligand 1, 2 expressions are decreased in the psoriatic epidermis. Arch Dermatol Res 2015; 307: 531-8. 